\title{
Effect of Environmental Salt Stress on Plants and the Molecular Mechanism of Salt Stress Tolerance
}

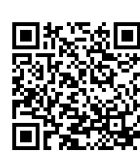

\author{
Wenbo Li*1 and Qing $\mathrm{Li}^{2}$ \\ ${ }^{1}$ Department of Medicine, Baylor College of Medicine, USA \\ ${ }^{2}$ Department of Environmental and Interdisciplinary Sciences, Texas Southern University, USA
}

Submission: December 07, 2017; Published: December 14, 2017

*Corresponding author: Wenbo Li, Department of Medicine, Baylor College of Medicine, 1 Baylor Plaza, MS185, Houston, Texas, 77030, USA, Tel: +1-713-798-3159; Email: wenbol@bcm.edu

\begin{abstract}
Abiotic environmental stresses can negatively impact plant growth and productivity. Soil salinity is one of the major environment limitations on plant yield and distribution. In this article, we review the damage that environmental salt stress has on plants and how plant tolerates salt stress on the molecular level. We discussed multiple signalling and listed some pathways with certain members identified elements and some of the several common salt tolerance determinants. We examined the effect of salinity stress on plant development by reviewing the intracellular metabolic processes with excessive $\mathrm{Na}^{+}$and $\mathrm{Cl}$. Salt stress affects every aspect of plant development, inhibits the growth of the plant, reduces the fresh weight and dry weight of root, stem, and leaf, decreases the expansion of leaf and changes the morphology of the leaf. It also suppresses photosynthesis and affects the ion homeostasis in plants, causing an imbalance of metabolism and oxidative stress. We also reviewed the molecular mechanism of plant salt stress tolerance in the form of the regulation of multiple physical and biochemical response, including the salt overly sensitive pathway, MAPK signal pathway, as well as other protein kinase and abscisic acid mediation. All in all, today's research elevates our understanding of plant salt-resistance mechanisms to an unprecedented level. And yet, further studies on more elements of the signalling pathways are needed to fully understand the mechanisms of plant stress tolerance.
\end{abstract}

Keywords: Plant Stress Tolerance; Abiotic Environment; Salinity; Salt Stress; Salt Overly Sensitive Pathway; Signal Transduction.

Abbreviations: SOS: Salt Overly Sensitive; ABA: Abscisic Acid; MAPK: Mitogen Activated Protein Kinase; MAPKK: Mitogen-Activated Protein Kinase Kinase; MAPKKK: Mitogen Activated Protein Kinase Kinase Kinase; CDPK: Calcium-Dependent Protein Kinase; RPK: Receptor-Like Protein Kinase.

\section{Introduction}

The abiotic environment is critical to the proper growth of plants, and abiotic stresses can negatively impact a plant's ability to thrive in a given environment. Salinity is one of the major environment limitations on plant yield and distribution. Saline soils cover about 3.1 percent of the land surface on earth, and this number goes up to 19.5 percent in irrigated land. Salinity in the soil does much damage to the plants and limits the growth and productivity. However, after thousands of millions of years of evolution, plants do not just retreat from these unfavourable environments. They adapted to the environment changes and developed their mechanism to cope with various types of stress.

\section{Effect of Salinity on Plants}

The toxic effect of salt stress includes several aspects. Uptake of excessive $\mathrm{Na}^{+}$and $\mathrm{Cl}^{-}$interferences many Intracellular metabolic processes. High salt concentration in the soil causes osmotic stress, which limits water uptake from soil [1]. High concentration of $\mathrm{Na}^{+}$nitration inhibits the absorption of nutrient elements. Meanwhile, ion toxicity and osmotic stress will also cause an imbalance of metabolism and oxidative stress [2]. As a whole plant, the toxic effect of salt stress includes the death of the plant or decrease of production capacity. Almost all plants show growth inhibition with salt stress. However, growth repression level and critical death concentration vary a lot among different plants. Salt stress affects almost all aspects of biological processes, including the growth of the plant, photosynthesis, protein synthesis and the metabolism of protein and phospholipid.

\section{Effect of Salt Stress on Plant Development}

Salt stress affects every aspect of plant development from germination to vegetative growth to reproductive growth. Sodium is an essential microelement for plant growth. Low concentration of salt stimulates the growth of the plant, increase the biomass of plants. However, the high concentration of plants inhibited the growth of the plant; reduce the fresh weight and dry weight of root, stem, and leaf $[3,4]$. Under low salt stress $(50 \mathrm{mM} \mathrm{NaCl})$, the fresh weight of Alhagi pseudoalhagi increased. However, the fresh weight decreased with a high concentration (100 and 200 $\mathrm{mM} \mathrm{NaCl}$ ). Excessive concentration of $\mathrm{NaCl}$ led to decrease in the biomass of cotton root, shot and leaf [5]. Most plants are sensitive to salinity in the soil. Salt stress can repress the growth of plant tissues and organs, shorten the flowering phase, accelerate the aging, death, and fall off of mature leaves [6]. 
Under high concentration salt stress, the most immediate response was the decrease of the expansion rate of the leaf surface [7]. Salt stress of $100 \mathrm{mM} \mathrm{NaCl}$ can shorten the growing region of sorghum leaf and decrease the speed of the cell growth in this region [8]. Salt stress [6] can also shorten the development of wheat main stem by 18 days. Moreover, the generation of the reproductive structure was brought forward. The flowering period was much earlier than untreated wheat, which indicates that salt stress can accelerate the development of the plant. Meanwhile, the number of phyllopodium and leaves decreased [9]. Salt stress can also decrease the deposit of dry materials and slow down the mature of the cell wall [10]. Similarly, salt stress can change the morphology of the leaf. Salt stress increased the thickness of leaf epidermal cells, mesophyll cell and palisade cell [11]. On the contrary, the thickness and intercellular space of leaf epidermal cells decreased due to salt stress caused by $\mathrm{NaCl}$ [12]. Potato leaf cells rounded and the cellular space and the number of chloroplasts decreased after salt stress [13]. Salt stress can also lead to the decrease in the surface area of the leaf and the opening of stomata [14].

\section{Salt Stress affects Plant Photosynthesis}

Salt stress affects plant photosynthesis in the short or long term. The short-term effect happens within a few hours to one or two days when exposed to salt stress. This reaction is crucial. Carbon assimilation stopped entirely during this period. However, the long-term effect happens after a few days when the plant was exposed to salt stress [6]. Due to the accumulation of salt in the leaf, the assimilation of carbon decreased obviously [15]. Many research shows that the photosynthesis was suppressed by salt stress $[14,16,17]$. However, some research shows that the rate of photosynthesis did not decrease under salt stress. The rate was even a little [14] higher than untreated condition [18]. The assimilation rate of carbon dioxide was slightly higher when Alhagi pseudoalhagi was treated with low salt. The assimilation rate was not affected when treated with $100 \mathrm{mM} \mathrm{NaCl}$. However, the assimilation rate of carbon dioxide dropped to $60 \%$ of the untreated plant when the salt treatment increased to 200 mM. Similarly, stomatal conductance was consistent with the assimilation rate of carbon dioxide. Moreover, the concentration of carbon dioxide in the intercellular space of plants was lower than the control group [18]. The carbon dioxide assimilation rate of Bidens parviflora increased under low concentration salt stress but decreased under high concentration salt stress [12].

Thylakoid structure of chloroplasts was disrupted when the plant was treated with salt treatment. The number and size of pellets of the plastid increased, and the starch content decreased $[19,20]$. Expansion of chloroplasts thylakoid membrane was observed in salt-treated potato [21]. The chloroplasts were found to be aggregated together in the leaves of salt treated tomato. Moreover, no grana and thylakoid structure were observed in chloroplasts [22]. The ultra-microstructure of salt treated Eucalyptus microcorys chloroplasts also changed. This will induce the production of numerous starch granules [23].

\section{Salt Stress affects the Ion Homeostasis in Plant}

Uptake of too much $\mathrm{NaCl}$ impaired the absorption of other nutrient ions, which will lead to the deficiency of $\mathrm{K}^{+}$. The increase of $\mathrm{NaCl}$ in the soil led to the increase of cellular $\mathrm{Na}^{+}$and $\mathrm{Cl}^{-}$and the decrease of $\mathrm{Ca}^{2+}, \mathrm{K}^{+}$, and $\mathrm{Mg}^{2+}[24,25]$. Salt stress can increase the contents of $\mathrm{Na}^{+}, \mathrm{Ca}^{2+}$, and $\mathrm{Cl}^{-}$in broad bean and decrease the $\mathrm{K}^{+} /$ $\mathrm{Na}^{+}$value [26]. The increase of $\mathrm{Na}^{+}$and $\mathrm{Cl}^{-}$in $U$. fasciata induced the accumulation of proline. It decreased the content of proline dehydrogenase (PDH) and soluble $\mathrm{Ca}^{2+}$ [27].

\section{The Molecular Mechanism of Plant Salt Stress Tolerance}

Plant salt stress tolerance involves the regulation of multiple physical and biochemical response. Many protein and metabolites are involved. This is a process with complicated gene network interaction. Moreover, different plants have different responses and adaptive mechanisms.

\section{Salt Overly Sensitive Pathway}

High level of salt stress can lead to $\mathrm{Na}^{+}$toxicity, and cause osmotic and oxidative stress, which will repress the growth and development of plants. Under salt stress, plant cells can efflux toxic $\mathrm{Na}^{+}$or isolate $\mathrm{Na}^{+}$in the vacuole to build new ion homeostasis. There Salt Overly Sensitive (SOS) gene (SOS1, SOS2, and SOS3) were isolated by using genetic methods. These three genes formed the critical SOS signal transduction pathway [28]. SOS1 is a $\mathrm{Na}^{+} /$ $\mathrm{H}^{+}$Channel protein with 10-12 trans membrane domains [29]. SOS1 may also be a $\mathrm{Na}^{+}$sensor. SOS2 is a Ser/ Thr protein kinase with a regulation domain at $\mathrm{C}$ terminal and a catalytic domain at $\mathrm{N}$ terminal [30]. SOS3 is a calcium binding protein Located in the cytoplasm. Salt treatment can transiently induce the Ca in the cytosol and this increase of $\mathrm{Ca}^{2+}$ can be perceived by SOS3 $[31,32]$. Then SOS3 will bind to an active SOS2 [33]. The activated SOS3SOS2 compound can phosphorylate SOS1 and pump $\mathrm{Na}^{+}$out of the cell [34]. Any mutation in this SOS pathway will increase plant sensitivity to salt. Overexpression of SOS1 or activation of SOS2 will improve plant salt tolerance $[35,36]$. Salt stress may also accumulate ABA in the cell. ABA signal can regulate SOS pathway through SOS2. Yeast two-hybrid found that SOS2 can bind to ABA Insensitive2 (ABI2) but not to ABI2 mutant abi2, Seedlings of abi2 are more sensitive to salt stress [37].

Salt stress can also induce oxidative stress. Research has shown that SOS1 can interact with the protein RCD1 (radical-induced cell death 1). RCD1 is a transcriptional regulator of Arabidopsis under oxidative stress. RCD1 is located in nucleus without any stress. However, with salt or oxidative stress, RCD1 is found in both nucleus and cytoplasm. Both sos1 and $r c d 1$ mutants are sensitive to oxidative and salt stress [38]. Too much cytosol $\mathrm{Na}^{+}$caused by salt stress will inhibit the absorption of potassium. All salt overly sensitive mutants lacked $\mathrm{K}^{+}$. By screening for the suppressors of sos3 [39], identified AtHKT1, which is a $\mathrm{Na}^{+}$transporter. It can transport $\mathrm{Na}^{+}$into the cell. The mutant of AtHKT1 can not only compensate the salt-sensitive phenotype of salt overly sensitive mutants. To reduce the cytoplasm sodium, transporting the sodium into the vacuole is a very effective strategy. AtNHX1 is 
a $\mathrm{Na}^{+} / \mathrm{H}^{+}$antiporter, which can transport $\mathrm{Na}^{+}$into vacuole. The expression of AtNHX1 can be induced by salt stress [40]. AtNHX2 and AtNHX5, which are homologs of AtNHX1, have the similar function in isolating the sodium in the plant vacuole [40-42].

\section{MAPK Signal Pathway}

The typical mitogen-activated protein kinase signalling pathway contains three members, which are MAPKKK, MAPKK and MAPK. Plant MAPK involves in development guidance, cell division, plant hormones, biotic and abiotic stress tolerance [43]. Salt stress can active MAPK protein SIMK (salt stress-induced MAPK).SIMKK can activate SIPK. Moreover, the activation can be enhanced by salt stress [44]. Salt stress can induce the expression and kinase activity of many MAPKs. AtMPK6 and AtMPK4 were activated under salt stress [45]. Phosphorylated upstream kinase AtMKK2 activated these two MAPKs. The mutant of $m k k 2$ was sensitive to salt stress [46]. Studies showed that MPK6 could bind to and phosphorylate SOS1. Studies have shown that phosphatidic acid binds to MPK6 after salt treatment [47]. MPK6 immuno precipitated by Arabidopsis thaliana under salt stress and increase in phosphatidic acid can increase the phosphorylation of SOS1 [48].

\section{Other Protein Kinase}

Protein kinases are essential signal transduction molecules in vivo. In addition to mitogen-activated protein kinases which play a significant role in salt stress signal transduction, many other protein kinases also played critical roles in the signal transduction network under salt stress.

Calcium-dependent protein kinase (CDPK) is a type of signal transduction factor that uses calcium concentration as an upstream signal. CDPK can sense calcium levels by binding to calcium ions and phosphorylate downstream proteins to convert calcium signals to phosphorylated signals. In Arabidopsis, AtCDPK1 and AtCDPK2 can be induced by both high salinity stress and drought stress [49]. One of the cloned proteins induced by salt stress, McCDPK, which is located in the nucleus, may be related to transcriptional regulation [50].

There is also a class of proteins located on the cell membrane, which can sense the outside signal and participate in intracellular signalling. These proteins are called receptor-like protein kinase (RPK). RPK1 is a receptor protein kinase located in the cell membrane of Arabidopsis that can be induced by high salinity or drought, and there is evidence that it is involved in plant salt [51]. AtGSK1 is also a protein kinase induced by salt stress. It is a glycogen synthase kinase (GSK). Over expression of At GSK1 can enhance the tolerance of Arabidopsis to salt stress [52]. Studies have shown that some salt-induced gene expression can be induced by AtGSK1 [53]. GRIK1, upstream kinase of SnRKs can phosphorylate SOS2 in vitro. Moreover, grik1 mutants are sensitive to high salt [54].

\section{Abscisic Acid (ABA) Mediated Salt Stress Signal}

ABA is a critical plant hormone that plays an important role in many aspects of plant growth and development. It is the best studied in plant drought stress tolerance. ABA also plays an important role in salt stress tolerance. Salt stress can induce the accumulation of ABA in some tissue. Taking corn as an example, the ABA concentration in root with salt stress can be ten times higher than that in leaves [55]. The shrinking of the cells due to osmotic stress also leads to the synthesis and accumulation of $A B A$, thereby regulating the osmotic stress that caused by oversalinity. ABA controls the cell's water balance by regulating the stomatal switch, and it also regulates the expression of genes that control osmotic synthesis [56,57]. ABA controls the closure of stomatal pore and the expression of downstream genes by modulating $\mathrm{Ca}^{2+}$ concentration [58,59].

Under salt stress, ABA can regulate osmotic stress corresponding gene and ion transporter. $\mathrm{ABA}$ activates stress response genes RD29A and KIN2 [60]. ABA can also induce AtPLC1 expression. A study of transgenic plants expressing the antisense strand of AtPLC1 found that At PLC1 expression was required for ABA to induce RD22, RD29A, and KIN2 [61]. Genetic screening of the RD29A::LUC reporter gene accelerated the screening of Arabidopsis abiotic stress mutants and ABA signaling pathway mutants [62]. Two of the mutants $\operatorname{los} 5$ and $\operatorname{los} 6$ attenuated the expression of stress response genes under salt stress, such as RD29A, COR15A, COR47, RD22, and P5CS. However, the expression of RD29A::LUC induced by salt stress in mutants could be restored to a reasonable level by the exogenous addition of ABA. They were found to be alleles of $A B A 3$ and $A B A 1$ respectively after cloning the two genes LOS5 and LOS6 [63,64]. ABA-deficient mutants los5 and los6 were both more tolerant to salt stress during germination. However, $\operatorname{los} 5$ was hypersensitive to salt stress during vegetative growth $[63,65]$. These results indicate that the expression of salt stress-responsive genes is mediated by ABA. Salt stress and ABA up-regulate the vacuolar $\mathrm{Na}^{+} / \mathrm{H}^{+}$transporter At NHX1, while the expression of AtNHX1 in ABA-deficient mutants aba21 and aba3-1 decreased but not in salt overly sensitive mutants. Mutant abi1-1 reduced ABA content and salt-induced AtNHX1 expression, whereas abi2-1 did not. AtNHX1 also contains ABRE elements, indicating that up regulation of AtNHX1 transcriptional level under salt stress is partly dependent on ABA synthesis and ABA signalling, a process that cannot be separated from ABI1 [66]. The QTL for salt tolerance in the germination period is very different from that of salt tolerance in the seedling stage, which also indicates that the mechanism of salt tolerance during the seedling stage is different from during germination stage $[67,68]$. The inhibition of salt stress on seed germination is mainly due to the inhibition of ABA by the stress [28].

\section{Studies on Other Salt Stress-Related Mutants}

In addition to studies on the above kinases and their pathways, many other mutants related to salt stress have also been found, yet their specific roles in salt stress tolerance remain unclear. $\operatorname{sos} 4$ and $\operatorname{sos} 5$ are two mutants obtained by screening salt hypersensitive mutants, which both exhibit significantly shorter root length than wild-type plants under salt stress. SOS4 encodes a pyridoxal kinase, which participates in the process of pyridoxal 5-phosphate formation. Pyridoxal 5-phosphate is an essential 
molecular chaperone of many enzymes. SOS4 is possibly involved in plant salt tolerance through producing pyridoxal 5- phosphate [66]. SOS5 encodes a cell surface adhesion protein. Its mutant sos5 is sensitive to salt due to failure to expand normally in the absence SOS5 [69]. Ren et al. used the salt stress sensitivity difference between Landsberg erecta (Ler), a salt and ABA sensitive ecotype of Arabidopsis thaliana, and Shakdara (Sha), an ecotype that is not sensitive to salt and ABA, to study salt sensitivity. They cloned a salt stress and ABA-induced gene RAS1 using quantitative trait locus technology (QTL). Overexpression of this protein enhanced plant sensitivity to ABA and salt stress [70]. Salt stress leads to the accumulation of reactive oxygen species (ROS) in plant cells. The plant has to response to neutralize the excessive ROS and many enzymes are involved in this process [71,72]. RSA3 is bi-functional protein, which can bind to and stabilize cytoskeleton to minimize the oxidative damage during salt stress in [73].

\section{Conclusion}

Salt is essential to any form of life on earth, be it animals, plants or bacteria. Modern biology suggests that it regulates blood flow and pressure in animals, mediates osmosis in plants and that it serves as critical messengers in cellular signal transduction. However, such vital elements are now known to become detrimental stresses to life if exceeding proper amounts. In fact, salt stress is one of the primary abiotic stresses that affect plant growth and decrease in crop yield. In this review, we discussed the damage that environmental salt stress can do to plants and how plant tolerates salt stress on the molecular level. Today's research elevates our understanding of plant salt-resistance mechanisms to an unprecedented level. We discussed multiple signaling pathways with certain identified elements and several common salt tolerance determinants. They make up part of the salt stress signaling network. However, there are other proteins, signal molecules and other mechanisms involved but not mentioned in this review, such as non-coding RNAs and epigenetics regulation. And yet, further studies on more elements of the signaling pathways are needed to fully understand the mechanisms of plant stress tolerance.

\section{References}

1. Munns R (2002) Comparative physiology of salt and water stress. Plant Cell Environ 25(2): 239250.

2. Chinnusamy V, Zhu J, Zhu JK (2006) Salt stress signaling and mechanisms of plant salt tolerance. Genet Eng 27: 141-177.

3. AliDinar HM, Ebert G, Ludders P (1999) Growth, Chlorophyll Content, Photosynthesis and Water Relations in Guava (Psidium guajava L.) Under Salinity and Different Nitrogen Supply. Garten bauwissens chaft 64(2): 54-59.

4. Chartzoulakis K, Klapaki G (2000) Response of two greenhouse pepper hybrids to $\mathrm{NaCl}$ salinity during different growth stages. Scientia Horticulturae 86(3): 247-260.

5. Meloni DA, Oliva MA, Ruiz HA, Martinez CA (2001) Contribution of proline and inorganic solutes to osmotic adjustment in cotton under salt stress. Journal of Plant Nutrition 24(3) 599-612.

6. Parida AK, Das AB (2005) Salt tolerance and salinity effects on plants: a review. Eco toxicol Environ Saf 60(3): 324-349.
7. Wang Y, Nii N (2000) Changes in chlorophyll, ribulose bisphosphate carboxylase-oxygenase, glycine betaine content, photosynthesis and transpiration in Amaranthus tricolor leaves during salt stress. The Journal of Horticultural Science and Biotechnology 75(6): 623-627.

8. Bernstein N, Lauchli A, Silk WK (1993) Kinematics and dynamics of sorghum (Sorghum bicolor L.) leaf development at various $\mathrm{Na} / \mathrm{Ca}$ salinities (I. Elongation growth). Plant Physiology 103(4): 1107-1114.

9. Grieve C, Francois L, Maas E (1994) Salinity affects the timing of phasic development in spring wheat. Crop science 34(6): 1544-1549.

10. Taleisnik E, Rodríguez AA, Bustos D, Erdei L, Ortega L, et al (2009) Leaf expansion in grasses under salt stress. J Plant Physiol 166(11): 11231140.

11. Longstreth DJ, Nobel PS (1979) Salinity effects on leaf anatomy: consequences for photosynthesis. Plant Physiol 63(4): 700-703.

12. Parida AK, Das A, Mittra B (2004) Effects of salt on growth, ion accumulation, photosynthesis and leaf anatomy of the mangrove, Bruguiera parviflora. Trees 18(2): 167-174.

13. Bruns S, Hecht Buchholz C (1990) Light and electron microscope studies on the leaves of several potato cultivars after application of salt at various development stages. Potato Research 33(1): 33-41.

14. Romero Aranda R, Soria T, Cuartero J (2001) Tomato plant-water uptake and plant-water relationships under saline growth conditions. Plant Sci 160(2): 265-272.

15. Munns R, Termaat A (1986) Whole-plant responses to salinity. Functional Plant Biology 13(1): 143-160.

16. Chaudhuri K, Choudhuri M (1997) Effects of short-term $\mathrm{NaCl}$ stress on water relations and gas exchange of two jute species. Biologia plantarum 40(3): 373-380.

17. Kao WY, Tsai HC, Tsai TT (2001) Effect of $\mathrm{NaCl}$ and nitrogen availability on growth and photosynthesis of seedlings of a mangrove species, Kandelia candel Druce. Journal of plant physiology 158(7): 841-846.

18. Kurban H, Saneoka H, Nehira K, Adilla R, Premachandra GS, et al. (1999) Effect of salinity on growth, photosynthesis and mineral composition in leguminous plant Alhagi pseudoalhagi (Bieb.). Soil science and plant nutrition 45(4): 851-862.

19. Hernandez J, Olmos E, Corpas F, Sevilla F, Del Rio L (1995) Salt-induced oxidative stress in chloroplasts of pea plants. Plant Science 105(2): 151-167.

20. Hernandez J, Campillo A, Jimenez A, Alarcon J, Sevilla F (1999) Response of antioxidant systems and leaf water relations to $\mathrm{NaCl}$ stress in pea plants. The New Phytologist 141(2): 241-51.

21. Mitsuya S, Takeoka Y, Miyake H (2000) Effects of sodium chloride on foliar ultrastructure of sweet potato (Ipomoea batatas Lam.) plantlets grown under light and dark conditions in vitro. Journal of plant physiology 157(6): 661-667.

22. Khavari Nejad R, Mostofi Y (1998) Effects of NaCl on photosynthetic pigments, saccharides, and chloroplast ultrastructure in leaves of tomato cultivars. Photosynthetica 35(1): 151-154.

23. Keiper F, Chen D, De Filippis L (1998) Respiratory, photosynthetic and ultrastructural changes accompanying salt adaptation in culture of Eucalyptus microcorys. Journal of Plant Physiology 152(4-5): 564-573.

24. Khan MA, Ungar IA, Showalter AM (1999) Effects of salinity on growth, ion content, and osmotic relations in Halopyrum mucronatum (L.) Stapf. Journal of Plant Nutrition 22(1): 191-204.

25. Khan MA, Ungar IA, Showalter AM (2000) Effects of sodium chloride treatments on growth and ion accumulation of the halophyte Haloxylon recurvum. Communications in Soil Science and Plant Analysis 31(1718): 2763-2774.

26. Gadallah M (1999) Effects of proline and glycinebetaine on Vicia faba responses to salt stress. Biologia plantarum 42(2): 249-257.

27. Lee TM, Liu CH (1999) Correlation of decreased calcium contents with proline accumulation in the marine green macroalga Ulva fasciata 
exposed to elevated $\mathrm{NaCl}$ contents in seawater. Journal of experimental botany 50(341): 1855-1862.

28. Chinnusamy V, Zhu JK (2003) Plant salt tolerance. Plant responses to abiotic stress 2(1): 241-270.

29. Shi H, Ishitani M, Kim C, Zhu JK (2000) The Arabidopsis thaliana salt tolerance gene SOS1 encodes a putative $\mathrm{Na}^{+} / \mathrm{H}^{+}$antiporter. Proceedings of the National Academy of Sciences USA 97(12): 6896-6901.

30. Liu J, Ishitani M, Halfter U, Kim CS, Zhu JK (2000) The Arabidopsis thaliana SOS2 gene encodes a protein kinase that is required for salt tolerance. Proceedings of the National Academy of Sciences 97(7): 3730-3734

31. Liu J, Zhu JK (1998) A calcium sensor homolog required for plant salt tolerance. Science 280(5371): 1943-1945.

32. Ishitani M, Liu J, Halfter U, Kim CS, Shi W, et al. (2000) SOS3 function in plant salt tolerance requires $\mathrm{N}$-myristoylation and calcium binding The Plant Cell 12(9): 1667-1678.

33. Halfter U, Ishitani M, Zhu JK (2000) The Arabidopsis SOS2 protein kinase physically interacts with and is activated by the calcium-binding protein SOS3. Proceedings of the National Academy of Sciences 97(7): 3735-3740.

34. Zhu JK (2003) Regulation of ion homeostasis under salt stress. Current opinion in plant biology 6(5): 441-445.

35. Shi H, Lee BH, Wu SJ, Zhu JK (2003) Overexpression of a plasma membrane $\mathrm{Na}+\mathrm{H}+$ antiporter gene improves salt tolerance in Arabidopsis thaliana. Nature biotechnology 21(1): 81-85.

36. Guo Y, Qiu QS, Quintero FJ, Pardo JM, Ohta M, et al (2004) Transgenic evaluation of activated mutant alleles of SOS2 reveals a critical requirement for its kinase activity and C-terminal regulatory domain for salt tolerance in Arabidopsis thaliana. The Plant Cell 16(2): 435 449.

37. Ohta M, Guo Y, Halfter U, Zhu JK (2003) A novel domain in the protein kinase SOS2 mediates interaction with the protein phosphatise 2C ABI2. Proceedings of the National Academy of Sciences 100(20): 11771-11776.

38. Katiyar Agarwal S, Zhu J, Kim K, Agarwal M, Fu X, et al. (2006) The plasma membrane $\mathrm{Na}+/ \mathrm{H}+$ antiporter SOS1 interacts with RCD1 and functions in oxidative stress tolerance in Arabidopsis. Proceedings of the National Academy of Sciences 103(49): 18816-18821.

39. Izhaki A, Swain SM, Tseng TS, Borochov A, Olszewski NE, et al. (2001) The role of SPY and its TPR domain in the regulation of gibberellin action throughout the life cycle of Petunia hybrida plants. The Plant Journal 28(2): 181-190.

40. Yokoi S, Quintero FJ, Cubero B, Ruiz MT, Bressan RA, et al. (2002) Differential expression and function of Arabidopsis thaliana NHX Na+/ $\mathrm{H}+$ antiporters in the salt stress response. The Plant Journal 30(5): 529-539.

41. Gaxiola RA, Rao R, Sherman A, Grisafi P, Alper SL, et al. (1999) The Arabidopsis thaliana proton transporters, AtNhx1 and Avp1, can function in cation detoxification in yeast. Proceedings of the National Academy of Sciences 96(4): 1480-1485.

42. Quintero FJ, Blatt MR, Pardo JM (2000) Functional conservation between yeast and plant endosomal $\mathrm{Na}+\mathrm{H}+$ antiporters 1 . FEBS letters 471(2-3): 224-228.

43. Morris PC (2010) Integrating lipid signalling, mitogen-activated protein kinase cascades and salt tolerance. New Phytologist 188(3): 640-643.

44. Kiegerl S, Cardinale F, Siligan C, Gross A, Baudouin E, et al. (2000) SIMKK, a mitogen-activated protein kinase (MAPK) kinase, is a specific activator of the salt stress-induced MAPK, SIMK. The Plant Cell 12(11): 2247-2258.

45. Ichimura K, Mizoguchi T, Yoshida R, Yuasa T, Shinozaki K (2000) Various abiotic stresses rapidly activate Arabidopsis MAP kinases
ATMPK4 and ATMPK6. The Plant Journal 24(5): 655-665.

46. Teige M, Scheikl E, Eulgem T, Dóczi R, Ichimura K, et al. (2004) The MKK2 pathway mediates cold and salt stress signaling in Arabidopsis. Molecular cell 15(1): 141-152.

47. Testerink C, Dekker HL, Lim ZY, Johns MK, Holmes AB, et al. (2004) Isolation and identification of phosphatidic acid targets from plants. The Plant Journal 39(4): 527-536.

48. Yu L, Nie J, Cao C, Jin Y, Yan M, et al. (2010) Phosphatidic acid mediates salt stress response by regulation of MPK6 in Arabidopsis thaliana. New Phytologist 188(3): 762-773.

49. Urao T, Yakubov B, Satoh R, Yamaguchi Shinozaki K, Seki M, et al. (1999) A trans membrane hybrid-type histidine kinase in Arabidopsis functions as an osmo sensor. The Plant Cell 11(9): 1743-1754.

50. Patharkar OR, Cushman JC (2000) A stress-induced calciumdependent protein kinase from Mesembryanthemum crystallinum phosphorylates a two-component pseudo-response regulator. The Plant Journal 24(5): 679-691.

51. Hong SW, Jon JH, Kwak JM, Nam HG (1997) Identification of a receptorlike protein kinase gene rapidly induced by abscisic acid, dehydration, high salt, and cold treatments in Arabidopsis thaliana. Plant physiology 113(4): 1203-1212.

52. Piao HL, Pih KT, Lim JH, Kang SG, Jin JB, et al. (1999) An Arabidopsis GSK3/shaggy-like gene that complements yeast salt stress-sensitive mutants is induced by $\mathrm{NaCl}$ and abscisic acid. Plant Physiology 119(4): 1527-1534.

53. Piao HL, Lim JH, Kim SJ, Cheong GW, Hwang I (2001) Constitutive overexpression of AtGSK1 induces $\mathrm{NaCl}$ stress responses in the absence of $\mathrm{NaCl}$ stress and results in enhanced $\mathrm{NaCl}$ tolerance in Arabidopsis. The Plant Journal 27(4): 305-314.

54. De Dios Barajas Lopez J, Moreno JR, Gamez Arjona FM, Pardo JM, Punkkinen M, et al. (2017) Upstream kinases of plant SnRKs are involved in salt stress tolerance. The Plant Journal.

55. Jia W, Wang Y, Zhang S, Zhang J (2002) Salt-stress-induced ABA accumulation is more sensitively triggered in roots than in shoots. Journal of Experimental Botany 53(378): 2201-2206.

56. Zhu JK (2002) Salt and drought stress signal transduction in plants. Annual review of plant biology 53(1): 247-273.

57. Wang ZY, Xiong L, Li W, Zhu JK, Zhu J (2011) The Plant Cuticle Is Required for Osmotic Stress Regulation of Abscisic Acid Biosynthesis and Osmotic Stress Tolerance in Arabidopsis. Plant Cell 23(5): 19711984.

58. Leung J, Giraudat J (1998) Abscisic acid signal transduction. Annual review of plant biology 49(1): 199-222.

59. Schroeder JI, Allen GJ, Hugouvieux V, Kwak JM, Waner D (2001) Guard cell signal transduction. Annual review of plant biology 52(1): 627658.

60. Wu Y, Kuzma J, Maréchal E, Graeff R, Lee HC, et al. (1997) Abscisic acid signaling through cyclic ADP-ribose in plants. Science 278(5346): 2126-2130.

61. Sanchez JP, Chua NH (2001) Arabidopsis PLC1 is required for secondary responses to abscisic acid signals. The Plant Cell 13(5): 1143-1154.

62. Ishitani M, Xiong L, Stevenson B, Zhu JK (1997) Genetic analysis of osmotic and cold stress signal transduction in Arabidopsis: interactions and convergence of abscisic acid-dependent and abscisic acid-independent pathways. The Plant Cell 9(11): 1935-1949.

63. Xiong L, Ishitani M, Lee H, Zhu JK (2001) The Arabidopsis LOS5/ABA3 locus encodes a molybdenum cofactor sulfurase and modulates cold stress-and osmotic stress-responsive gene expression. The Plant Cell 13(9): 2063-2083.

64. Xiong L, Lee H, Ishitani M, Zhu JK (2002) Regulation of Osmotic Stressresponsive Gene Expression by theLOS6/ABA1 Locus in Arabidopsis. 
Journal of Biological Chemistry 277(10): 8588-8596.

65. Xiong L, Schumaker KS, Zhu JK (2002) Cell signaling during cold, drought, and salt stress. The plant cell 14(1): 165-183.

66. Shi H, Zhu JK (2002) Regulation of expression of the vacuolar $\mathrm{Na}+/$ $\mathrm{H}+$ antiporter gene AtNHX1 by salt stress and abscisic acid. Plant molecular biology 50(3): 543-550.

67. Quesada V, Garcia Martınez S, Piqueras P, Ponce MR, Micol JL (2002) Genetic architecture of $\mathrm{NaCl}$ tolerance in Arabidopsis. Plant physiology 130(2): 951-963.

68. Mano Y, Takeda K (1997) Mapping quantitative trait loci for salt tolerance at germination and the seedling stage in barley (Hordeum vulgare L.). Euphytica 94(3): 263-272.

69. Shi H, Kim Y, Guo Y, Stevenson B, Zhu JK (2003) The Arabidopsis SoS5 locus encodes a putative cell surface adhesion protein and is required for normal cell expansion. The Plant Cell 15(1): 19-32.

70. Ren Z, Zheng Z, Chinnusamy V, Zhu J, Cui X, et al. (2010) RAS1, a quantitative trait locus for salt tolerance and ABA sensitivity in Arabidopsis. Proceedings of the National Academy of Sciences 107(12): 5669-5674.

71. Nxele X, Klein A, Ndimba B (2017) Drought and salinity stress alters ROS accumulation, water retention, and osmolyte content in sorghum plants. South African Journal of Botany 108: 261-266.

72. Hamada AbdElgawad GZ, Hegab MM, Pandey R, Asard H, Abuelsoud W (2016) High salinity induces different oxidative stress and antioxidant responses in maize seedlings organs. Frontiers in plant science 7: 276

73. Li W, Guan Q, Wang ZY, Wang Y, Zhu J (2013) A bi-functional xyloglucan galactosy ltransferase is an indispensable salt stress tolerance determinant in Arabidopsis. Molecular plant 6(4): 1344-1354.

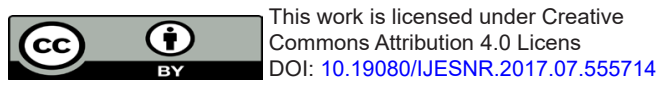

